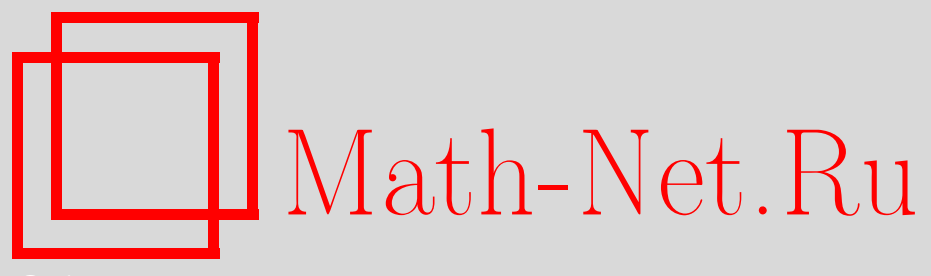

Е. А. Горин, Фрагменты научной биографии Д. А. Райкова: гармонический анализ, УМН, 2006, том 61, выпуск $5,157-172$

DOI: https://doi.org/10.4213/rm5325

Использование Общероссийского математического портала Math-Net.Ru подразумевает, что вы прочитали и согласны с пользовательским соглашением http://www . mathnet.ru/rus/agreement

Параметры загрузки:

IP : 34.239 .49 .27

26 апреля 2023 г., 11:51:11

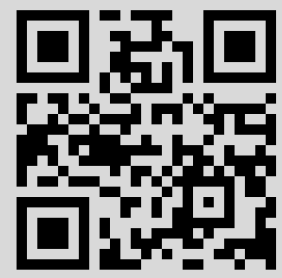




\section{Фрагменты научной биографии Д. А. Райкова: гармонический анализ}

\section{Е. А. Горин}

Приводятся основные биографические сведения о Д.А. Райкове, его научных интересах, публикациях, ближайших учениках. Дано доступное описание научных достижений Д. А. Райкова в абстрактном гармоническом анализе и некоторых направлений их дальнейшего развития.

Библиография: 22 названия.

\section{СОДЕРЖАНИЕ}

$\S 1$. Предисловие .......................................... 157

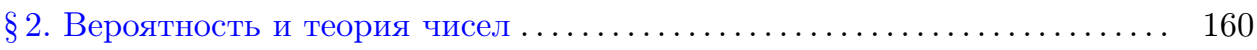

$\S 3$. Положительно-определенные функции на группах ............... 162

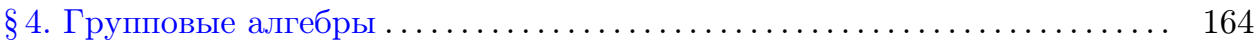

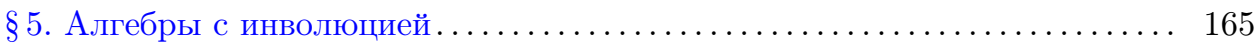

$\S 6$. Дополнительные примеры . . . . . . . . . . . . . . . . . . . . . 166

Список литературы ........................................ 171

\section{§ 1. Предисловие}

К условным ученикам Д. А. Райкова можно отнести большинство русскоговорящих математиков XX века, поскольку многие из нас учились по книгам, написанным его рукой: учебникам, монографиям, переводам, снабженным его предисловиями и комментариями.

В свое время практически все аспиранты-математики использовали двухтомник алгебры Ван дер Вардена и задачник Пойа и Сегё. В 50-е годы, когда научные интересы самого Д.А. переместились в область топологических векторных пространств, он сам и его ученики перевели на русский язык несколько удачных руководств по этому предмету. Д.А. и сам написал очень полезное введение в теорию векторных пространств.

Многие учились по знаменитой статье в УМН и книге трех авторов (И. М. Гельфанд, Д.А. Райков, Г.Е. Шилов) по банаховым алгебрам и по монографии Люмиса по абстрактному гармоническому анализу, и нет числа тем, кто обращался к 1000-страничному двухтомнику Б.Н. Делоне и Д. А. Райкова по аналитической геометрии. Целые поколения студентов 
учились по оригинальным учебникам Д.А. Райкова по математическому анализу (ориентированным на математические факультеты пединститутов).

Эти сочинения, выходившие на протяжении более 50 лет, в заметной мере сохраняют актуальность и по сей день. Нельзя не отметить и большой вклад, который внес Д.А. в формирование современного математического русского языка.

О личной жизни Д.А. написано мало. Краткие биографические данные дает "Математика в СССР за сорок лет" [1], где в первом томе на с. 579-580 написано (самим Д.А., на что указывает справочник) буквально следующее:

Райков Дмитрий Абрамович, род. 11 нояб. (29 окт.) 1905 г. в г. Одессе, окончил Московский ун-т (1929), д-р физ.-мат. наук (1941), професcop (1950), в 1933-1935 работал в Воронежском ун-те, в 1935-1943в Гостехиздате, в 1938-1948 - в Матем. ин-те АН, в 1949-1952 - в Костромском пед. ин-те, в 1952-1956 - в Шуйском пед. ин-те, с 1957 работает в Московском гос. пед. ин-те.

Эти произнесенные скороговоркой даты многое (но не все) скажут людям старшего и среднего поколения. Сам Д.А. говорил, что одесская действительность начала века оказала сильное влияние на его формирование. В войну Д.А. оказался в ополчении, имел правительственные награды, был демобилизован по ранению.

Из Шуи Д.А. пригласил в Москву П. С. Новиков, который тогда заведовал кафедрой математического анализа в Московском пединституте им. В. И. Ленина (бывшие Высшие женские курсы, ныне - МПГУ). Петр Сергеевич хорошо знал Райкова. Время было сложное, однако П.С. был масштабной личностью, и в результате Д.А. плодотворно проработал в Московском пединституте до конца жизни, читал спецкурсы и руководил аспирантами в МГУ, активно сотрудничал в математических редакциях.

Журнал "Успехи математических наук" неоднократно печатал научные статьи и заметки Д.А., однако (по причинам, не всем понятным) никогда не писал о нем лично. К 75-летию Д.А. появилась посвященная ему короткая статья в "Математике в школе", составленная его сотрудниками по кафедре - П. В. Семеновым и В. П. Федоровой, а через несколько месяцев там же появился и некролог (Д. А. Райков умер в самом конце декабря 1980 г.). Значительно позже, в 2004 г., в МПГУ вышел сборник [2], посвященный 70-летию кафедры математического анализа (этой кафедрой до П. С. Новикова заведовали А. Я. Хинчин и Ф. Р. Гантмахер). В сборнике есть короткие статьи с воспоминаниями о Д.А., одна, написанная В.П. Федоровой, и вторая, написанная В.С. Сековановым, одним из последних прямых учеников Д.А. ${ }^{1}$ Вообще среди многочисленных прямых учеников Д.А. по общему мнению прежде всего надо назвать следующих: Л. В. Апарина, И. А. Березанский, Д. В. Ботнару, Б. С. Брудовский, М. М. Буняев, Ю. Н. Владимирский, В. Б. Гисин, А. С. Дынин, П. С. Кендеров, В. Л. Левин, А. Е. Мерзон, А. Х. Назиев, И. И. Подгорная, В. С. Ретах, В. С. Секованов, В. П. Федорова, В. Е. Холодовский.

\footnotetext{
${ }^{1}$ К настоящему времени в МПГУ вышел сборник, приуроченный к 100-летию со дня рождения Д. А. Райкова, и проведены некоторые другие мероприятия, связанные с этим событием.
} 
Я добавил бы к этому, что многие имели полезные неформальные контакты с Д.А. ${ }^{2}$ В середине $50-$ х годов Д.А. читал спецкурсы по топологическим векторным пространствам в МГУ. Мы с Б. С. Митягиным, хотя и слушали лекции Д.А., к тому моменту уже были тесно связаны с Г. Е. Шиловым и решили одну задачку по векторным пространствам, которую Г.Е. предложил на своем спецкурсе. Из редакции УМН наша заметка попала к Д.А., он добавил к ней текст, по объему сравнимый с исходным и привел ее к "нормальному виду" (мы по неопытности не сообразили пригласить его в соавторы, хотя и по содержанию добавления Райкова, пожалуй, не уступали исходному тексту). Так появилась наша первая публикация.

Д.А. был рецензентом моей дипломной работы, а через 12 лет - официальным оппонентом по докторской диссертации. До самого конца у меня сохранялись с ним ровные отношения. Сейчас я "завершаю свою трудовую деятельность" на той же кафедре, на которой все последние годы работал Д. А. Райков, о чем на кафедре напоминает его портрет (кстати, Д.А. когда-то приглашал меня перейти на эту кафедру из МГУ, однако это состоялось гораздо позже, и вместе нам работать не довелось).

Что касается упомянутых выше статей о Д. А. Райкове, то стоит отметить, что они написаны эмоционально, с любовью, однако (по законам жанра) математической информации в них мало. K тому же эти статьи опубликованы в мало доступных изданиях (которые к тому же не реферируются и не переводятся).

Вместе с тем, собственно математические работы Д.А. и его учебники описаны лучше. Есть статьи в "Математике в СССР" за 30 лет [3], за 40 лет [1; т. 1], список публикаций до 1967 г. включительно во 2-м томе "Математики в СССР 1958-1967" [4] (1-й том со статьями не вышел), а описание всего остального можно при желании найти в РЖ "Математика" или Mathematical Reviews. 3aметная часть работ Д.А. отражена в учебниках теории вероятностей, теории чисел, в книгах по абстрактному гармоническому анализу и теории топологических векторных пространств.

В этой статье я опишу лишь некоторые математические сюжеты, с которыми имел дело Д. А. Райков и которые лично мне несколько ближе, чем остальное. Например, я совсем не буду касаться его учебников и научно-методических открытий. Работам Д. А. Райкова по топологическим векторным пространствам и элементам теории категорий должны быть посвящены отдельные тексты. Вместе с тем, я упомяну некоторые известные мне результаты других авторов (в том числе и свои), достаточно тесно связанные с исследованиями Д.А.

Разумеется, я не претендую ни на полноту, ни на объективность, однако я постараюсь быть понятным. ${ }^{3}$

${ }^{2} \mathrm{O}$ чем бы я ни писал, я пишу о себе.

${ }^{3}$ Я благодарю А.Е. Мерзона, В.С. Ретаха и В.П. Федорову за предоставленную мне обширную библиографическую информацию, уточнение списка ближайших учеников Д. А. Райкова и ряд замечаний, которые я постарался учесть. Я приношу искренние извинения тем из учеников Д. А. Райкова, которых (быть может) я здесь не назвал. 


\section{§ 2. Вероятность и теория чисел}

Теорией вероятностей Д.А. Райков начал заниматься под руководством А.Я. Хинчина. Быть может, от Хинчина он воспринял и интерес к теории чисел. В дальнейшем он перешел к гармоническому анализу, однако и в теории вероятностей, и в теории чисел Д.А. интересовали такие задачи, которые непосредственно связаны с формировавшимися в 30-е годы общими понятиями (абстрактного) гармонического анализа.

В соответствии с аксиоматикой А. Н. Колмогорова скалярная случайная величина $\xi$ - это измеримое отображение $\xi: \Omega \rightarrow \mathbb{R}$ пространства с вероятностной мерой $\{\Omega, \mathrm{P}\}$ (так что $\mathrm{P}(\Omega)=1$ ) в вещественную ось $\mathbb{R}$.

Распределением случайной величины $\xi$ называется борелевская мера $\mu$ на $\mathbb{R}$, которая на стрелках $[a, b)$ определяется равенством

$$
\mu([a, b))=\mathrm{P}(\{\omega \in \Omega \mid a \leqslant \xi(\omega)<b\}) .
$$

Функция распределения $F_{\xi}=F_{\xi}(t)$ определяется равенством $F_{\xi}(t)=$ $\mu((-\infty, t))$. Заметим, что при таком определении функция $F_{\xi}$ непрерывна слева (а если, как зачастую делают, включить правый конец $t$, получится непрерывность справа).

Преобразование Фурье меры $\mu$ называется характеристической функцией распределения $\xi$. Таким образом, при вещественных $x$ характеристическая функция $f(x)$ может быть представлена несколькими способами:

$$
\begin{aligned}
f(x) & =\mathrm{M}\left(e^{i x \xi}\right) \\
& =\int_{\Omega} e^{i x \xi(\omega)} \mathrm{P}(d \omega) \\
& =\int_{\mathbb{R}} e^{i x t} \mu(d t) \\
& =\int_{\mathbb{R}} e^{i x t} d F_{\xi}(t),
\end{aligned}
$$

где $\mathrm{M}$ - знак математического ожидания, две следующие строчки включают интегралы Лебега, а последняя - интеграл Стилтьеса. Имеется внутреннее описание характеристических функций, на чем мы остановимся ниже.

Связь между распределениями и отвечающими им характеристическими функциями проще и прозрачнее, чем связь между общими мерами ограниченной вариации и их преобразованиями Фурье (хотя и в случае вероятностных мер эту связь нельзя назвать тривиальной). Например, если характеристическая функция $f(x)$ продолжается до аналитической в диске $|z|<a$ комплексной плоскости $\mathbb{C}$, то для распределения справедливо неравенство

$$
\int_{\mathbb{R}} e^{b|t|} \mu(d t)<\infty
$$

при всех $b<a$ (обратное очевидно), и тогда $f$ продолжается до аналитической в полосе $|\operatorname{Im} z|<a$. В частности, $f$ продолжается до целой функции тогда и только тогда, когда указанный интеграл сходится при всех $b>0$. 
Упорядоченная пара $\xi_{1}, \xi_{2}$ скалярных случайных величин естественно порождает меру в $\mathbb{R}^{2}$, которая называется совместным распределением этих величин. В рамках колмогоровской аксиоматики случайные величины $\xi_{1}, \xi_{2}$ независимы, если их совместное распределение есть прямое произведение $\mu_{1} \times \mu_{2}$ распределений компонент. В этом случае распределение суммы $\xi_{1}+\xi_{2}$ есть свертка $\mu_{1} * \mu_{2}$ и, следовательно, характеристическая функция суммы есть произведение характеристических функций компонент, $\widehat{\mu}_{1} \cdot \widehat{\mu}_{2}$.

Из очень простых соображений вытекает, что компоненты свертки распределений далеко не произвольны. В частности, привлекая теорему Фубини, получаем, что компоненты целой характеристической функции сами будут целыми. Уточнение этого наблюдения приводит к теореме Крамера: компоненты нормального распределения нормальны. Д. А. Райков в 1938 г. установил, что аналогичная теорема имеет место и для распределения Пуассона. Поскольку характеристическая функция распределения Пуассона,

$$
f_{\lambda}(x)=e^{\lambda\left(e^{i x}-1\right)}
$$

выглядит заметно сложнее, чем $e^{-x^{2}}$, и теорема Райкова выглядит сложнее теоремы Крамера.

Эти результаты породили целые направления. С одной стороны, арифметика вероятностных распределений в $\mathbb{R}^{n}$ благодаря дальнейшим работам Ю. В. Линника, И. В. Островского, Г. П. Чистякова (см., в частности, [5] [6]) и других превратилась в глубокую и далеко продвинутую теорию с обилием тонких аналитических результатов. Заметим, что изложение результатов, полученных в этом направлении до конца 70-х годов, имеется в монографии Лукача [7] (и примечаниях переводчика В.М.Золотарёва). С другой стороны, начиная с П. Леви стали заниматься вероятностными распределениями на других группах, например, на окружности $\mathbb{T}$. В частности, Марцинкевич обнаружил, что для группы $\mathbb{T}$ нет аналога теоремы Крамера, а существенно позже Г.М. Фельдман показал, что наличие в группе топологической копии группы $\mathbb{T}$ - единственное препятствие к нормальности компонент нормального распределения. Многочисленные результаты такого типа изложены в его монографии [8].

Относящиеся к теории чисел исследования Д. А. Райкова связаны с доказательством асимптотического закона распределения простых чисел ${ }^{4}$. Хотя формулировку PNT (и далеко идущих обобщений) знал Гаусс, очень близки к доказательству были П.Л. Чебышёв и Риман, первое аккуратное доказательство нашли Адамар и Валле Пуссен. Один из центральных моментов доказательства составляет неравенство $\zeta(1+i t) \neq 0, t \in \mathbb{R}$. Кстати, широко известное доказательство этого факта, основанное на тригонометрическом неравенстве, придумал Мертенс. Ниже я расскажу, как доказать, что $\zeta(1+i t) \neq 0$, используя только весьма общие соображения, связанные с понятием положительной определенности.

Пусть $\pi(x)$ - количество простых $p$, меньших, чем $x$. При натуральном $n$ положим $\Lambda(n)=\log p$, если $n$ есть степень какого-нибудь простого числа $p$ и

\footnotetext{
${ }^{4} \mathrm{~B}$ англоязычных текстах используется (по-моему, вполне вразумительная) аббревиатура PNT (от Prime Number Theorem), и мы будем использовать это сокращение.
} 
$\Lambda(n)=0$ во всех остальных случаях (функция Мангольдта). Функция

$$
\psi(x)=\sum_{n<x} \Lambda(n)
$$

называется функцией Чебышёва. Еще Чебышёв знал, что соотношение $\psi(x) \sim x$ при $x \rightarrow \infty$ эквивалентно $\mathrm{PNT}$, т.е. соотношению $\pi(x) \sim x / \log x$. Легко проверяется соотношение

$$
-\frac{\zeta^{\prime}(s)}{\zeta(s)}=s \int_{1}^{\infty} \frac{\psi(u) d u}{u^{s+1}} .
$$

Поэтому PNT получится, если по асимптотике преобразования Лапласа меры научиться определять асимптотику меры на бесконечности. Теоремы такого типа называются тауберовыми, и Д.А. занимался обобщением так называемой тауберовой теоремы Винера-Икеары. Законченную форму это обобщение приобрело существенно позже в работе Деланжа. Укажем, кстати, на очень содержательный обзор тауберовой теории [9] (не включающий, правда, алгебраический подход Гельфанда-Шилова).

Есть две публикации Д.А. конца 30-х годов, посвященные теоремам Л.Г. Шнирельмана о плотности арифметической суммы двух множеств натуральных чисел.

Особняком стоит написанная в те же годы небольшая заметка Д.А. о локальном равномерном приближении, содержащая, казалось бы, очень простое наблюдение: скорость приближения (полиномами) зависит не только от гладкости приближаемой функции, но и от величины промежутка, на котором происходит приближение. В середине 60-х годов это наблюдение стало исходным фактом красивой теории локальных приближений, построенной Ю.А. Брудным (см., в частности, [10]).

\section{§ 3. Положительно-определенные функции на группах}

Работа Д.А. по гармоническому анализу началась в очень интересный исторический период ${ }^{5}$. Незадолго до этого в самостоятельную дисциплину оформилась общая топология, появилась теория банаховых пространств, спектральный анализ в гильбертовом пространстве становился языком новой физики, была построена структурная теория и теория двойственности для локально компактных абелевых групп, были получены фундаментальные результаты в теории коммутативных банаховых алгебр, начала развиваться теория бесконечномерных представлений.

Я буду считать, что читатель имеет некоторое представление о локально компактных абелевых группах (мера Хаара, теорема двойственности Понтрягина) и основных понятиях, касающихся банаховых алгебр. Я не буду указывать источники, поскольку речь идет об "общих местах", однако по ходу дела некоторые факты я все-таки напомню.

\footnotetext{
53десь речь идет не о “международном положении”, которое тогда было, пожалуй, как раз "страшно интересным".
} 
Пусть $X$ - локально компактная абелева группа (записываемая аддитивно). В данном контексте непрерывный гомоморфизм $\chi: X \rightarrow \mathbb{T}$ называется характером группы $X$. Относительно поточечного умножения характеры сами составляют абелеву группу. Снабженная топологией равномерной сходимости на компактах эта группа называется двойственной и обозначается $\widehat{X}$ или $X^{\wedge}$ (мы для краткости обычно будем писать $G$ вместо $\widehat{X}$ ). Теорема двойственности Понтрягина ${ }^{6}$ устанавливает, что естественное непрерывное отображение $X \rightarrow\left(X^{\wedge}\right)^{\wedge}$ - топологический изоморфизм.

Формула $\chi_{\lambda}(x)=e^{i \lambda x}$ устанавливает естественное (в обыденном смысле слова) биективное соответствие между $\widehat{\mathbb{R}}$ и $\mathbb{R}$, а формулы $z \rightarrow z^{n}$ и $n \rightarrow z^{n}$ - между $\widehat{\mathbb{T}}$ и $\mathbb{Z}($ и $\widehat{\mathbb{Z}}$ и $\mathbb{T})$. Основные теоремы об интеграле Фурье на группах были установлены (вслед за построением Понтрягиным теории характеров) Д. А. Райковым и М. Г. Крейном. Меры Хаара на группах $X$ и $G$ можно согласовать так, чтобы в предположении, что обе функции $f$ и $\widehat{f}$ непрерывны и суммируемы, соотношения

$$
\widehat{f}(\chi)=\int_{X} \chi(x) f(x) d x \quad \text { и } \quad f(x)=\int_{G} \overline{\chi(x)} \widehat{f}(\chi) d \chi
$$

стали эквивалентными. Например, при $X=\mathbb{R}$ с этой целью перед знаком интеграла ставят $1 /(2 \pi)^{1 / 2}$.

Борелевские меры конечной полной вариации на $X$ с вариацией в качестве нормы и сверткой в качестве умножения составляют комплексную банахову алгебру (с единицей $\mathbb{I}$ ), которая обозначается $M(X)$. Конус положительных мер обозначается $M^{+}(X)$. Замкнутый идеал мер, абсолютно непрерывных по мере Хаара, естественно отождествляется с $L^{1}(X)$. Совпадение $M=L^{1}$ имеет место тогда и только тогда, когда группа $X$ дискретна.

Пусть $f: X \rightarrow \mathbb{C}-$ (не обязательно непрерывная) комплексная функция на $X$. Функция $f$ называется положительно-определенной, если для каждого конечного набора точек $\left\{x_{1}, x_{2}, \ldots, x_{n}\right\} \subset X$ и набора комплексных чисел $\lambda_{1}, \lambda_{2}, \ldots, \lambda_{n}$ имеет место неравенство

$$
\sum_{p, q=1}^{n} f\left(x_{p}-x_{q}\right) \lambda_{p} \bar{\lambda}_{q} \geqslant 0 .
$$

Класс всех положительно-определенных функций на группе $X$ будем обозначать $\mathrm{PD}(X)$.

Каждый характер принадлежит $\mathrm{PD}(X)$. В частности, это верно для непрерывных характеров. Поэтому $f \in \mathrm{PD}(X)$, если

$$
f(x)=\widehat{\mu}(x) \stackrel{\text { def }}{=} \int_{G} \chi(x) \mu(d \chi), \quad \text { где } \quad \mu \in M^{+}(G) .
$$

Тот факт, что каждая непрерывная положительно-определенная функция допускает такое представление, принято называть теоремой Бохнера. Хьюитт и Росс в [11; т. II, §33] указывают, что при $X=\mathbb{Z}$ эту теорему установил

\footnotetext{
${ }^{6}$ У Понтрягина было оказавшееся несущественным (ван Кампен) дополнительное предположение о счетной базе топологии.
} 
Герглотц в 1911 г. (в связи с работами Каратеодори и Тёплица). В начале 30 -х годов Бохнер получил ее для $\mathbb{R}^{n}$, а общий случай почти одновременно А. Вейль, Райков и А. Я. Повзнер.

В теореме Бохнера нет ничего мистического. В принципе дело обстоит так. Непрерывная положительно-определенная функция $f$ порождает по формуле

$$
\psi(g)=\int_{X} g(x) f(x) d x
$$

непрерывный функционал на $L^{1}(X)$, который естественно переносится на $\left(L^{1}\right)^{\wedge}$ и оказывается там положительным: $\psi(\widehat{g}) \geqslant 0$, если $\widehat{g} \geqslant 0$, после чего остается применить теорему Рисса (практически так действовали все, причем в одномерном случае (когда $X=\mathbb{R})$, который, как оказывается, мало чем отличается от общего, рассуждение придумал $\Phi$. Рисс).

Заметим, что характеристические функции отличаются от общих непрерывных положительно-определенных функций (на локально компактных абелевых группах) только условием нормировки $f(0)=1$.

Отметим, что некоторые свойства положительно-определенных функций, например, $|f(x)| \leqslant f(0)$ или $f(-x)=\overline{f(x)}$, легко следуют из определения, тогда как ряд других удобнее всего выводить из теоремы Бохнера (если заранее ничего не известно относительно непрерывности, группу следует считать дискретной). В частности, так проще всего доказать неравенство М. Г. Крейна:

$$
|f(x)-f(y)|^{2} \leqslant 2 f(0)\{f(0)-\operatorname{Re} f(x-y)\},
$$

из которого, в частности, вытекает, что $f$ равномерно непрерывна, если $\operatorname{Re} f$ непрерывна в точке $x=0$. Отметим еще, что для $f \in \operatorname{PD}(X)$ непрерывность в нуле вытекает из непрерывности сужения на довольно узкие множества (например, объединение координатных осей на плоскости или гиперповерхность положительной кривизны), и это получается при помощи следующего обобщения неравенства Крейна [12]:

$$
\left|f\left(\sum_{k=1}^{n} x_{k}\right)-f\left(\sum_{k=1}^{n} y_{k}\right)\right|^{2} \leqslant 2 n f(0) \sum_{k=1}^{n}\left\{f(0)-\operatorname{Re} f\left(x_{k}-y_{k}\right)\right\} .
$$

Отметим еще один очевидный, но очень полезный факт. Пусть $L^{1}(X)-$ совокупность суммируемых по мере Хаара функций на локально компактной абелевой группе с двойственной группой $G$, и пусть $f \in C(X) \cap L^{1}(X)$. В таком случае $f \in \mathrm{PD}(X)$ тогда и только тогда, когда имеет место неравенство

$$
\widehat{f}(\chi) \stackrel{\text { def }}{=} \int_{G} \chi(x) f(x) d x \geqslant 0 \quad \text { для всех } \quad \chi \in G
$$

(интеграл по мере Хаара).

\section{§ 4. Групповые алгебры}

Говоря о банаховых алгебрах, мы имеем в виду алгебры $A$ над полем $\mathbb{C}$ комплексных чисел. Обычно предполагается, что $\|a b\| \leqslant\|a\| \cdot\|b\|$ для всех $a, b \in A$. 
Если в алгебре есть единица II по умножению, то дополнительно предполагается, что $\|\mathbb{I}\|=1$. В банаховой алгебре есть норма, эквивалентная данной и удовлетворяющая этим условиям. Предположение о наличии единицы обычно (но не всегда) не является обременительным, хотя в случае групповых алгебр (о которых пойдет речь) наличие "естественной" единицы влечет за собой дискретность группы.

Для коммутативных банаховых алгебр $A$ с единицей очень важно описание гомоморфизмов $\varphi: A \rightarrow \mathbb{C}$, для которых $\varphi(\mathbb{I})=1$. Все такие гомоморфизмы непрерывны и, более того, $\|\varphi\|=1$. В слабой топологии сопряженного пространства они составляют компакт, расположенный на единичной сфере. $\operatorname{ker}(\varphi)$ - максимальный идеал, и других максимальных идеалов нет (И. М. Гельфанд). Поэтому $\operatorname{Spec}_{A}(a)=\{\varphi(a)\}$.

Такое описание спектра остается полезным и для некоммутативных алгебр $B$, так как каждый элемент $b \in B$ включается в такую коммутативную банахову подалгебру $A \subset B$ с той же единицей, что $\operatorname{Spec}_{B}(b)=\operatorname{Spec}_{A}(b)$.

Если $X$ - локально компактная абелева группа, то $A=L^{1}(X)$ со сверткой в качестве умножения называется групповой алгеброй группы $X$. Гельфанд и Райков среди прочего установили, что комплексные гомоморфизмы этой алгебры исчерпываются списком $\varphi(f)=\widehat{f}(\chi), \chi \in G$, и что эта алгебра тогда и только тогда обладает единицей по свертке, когда группа $X$ дискретна (и тогда $\left.L^{1}(X)=M(X)\right)$.

Таким образом, возникает топологическая эквивалентность между максимальными идеалами групповой алгебры $L^{1}(X)$ и группой характеров $G$. Эта эквивалентность в конечном счете позволила Д.А. вывести основные факты понтрягинской теории из гельфандовской интерпретации преобразования Фурье на группах. Кроме того, Д.А. получил первые нетривиальные результаты о структуре пространства максимальных идеалов алгебры $M(X)$, в связи с чем он изобрел так называемые системы Райкова, которые в дальнейшем широко применялись в других работах по гармоническому анализу.

Отчасти эти исследования завершил Ю. А. Шрейдер. Исследования Райкова и Шрейдера описаны в [13; гл. 4, 5]. Среди более поздних работ можно отметить работы Ж. Тейлора (см., в частности, [14]), который объяснил наличием аналитической структуры в порциях пространства максимальных идеалов алгебры $M(X)$ эффект Винера-Питта, а также вычислил когомологии такого пространства. Хотя многое прояснилось, алгебра $M(X)$ (в том числе простейшие примеры, скажем, $M(\mathbb{T}))$ остается весьма интересным и загадочным объектом.

\section{§ 5. Алгебры с инволюцией}

Пусть $A$ - алгебра с единицей $\mathbb{I}$ над полем $\mathbb{C}$ комплексных чисел. Инволюцией называется такое отображение $x \rightarrow x^{*}$ алгебры на себя, что

$$
\begin{aligned}
& \left(x^{*}\right)^{*}=x \text { для всех } x \in A ; \\
& \left(\lambda_{1} x_{1}+\lambda_{2} x_{2}\right)^{*}=\bar{\lambda}_{1} x_{1}^{*}+\bar{\lambda}_{2} x_{2}^{*} \text { для всех } \lambda_{1}, \lambda_{2} \in \mathbb{C} \text { и } x_{1}, x_{2} \in A ; \\
& \left(x_{1} x_{2}\right)^{*}=x_{2}^{*} x_{1}^{*} \text { для всех } x_{1}, x_{2} \in A .
\end{aligned}
$$


Как отмечается в [13], в контексте банаховых алгебр алгебры с инволюцией впервые появились в работе Гельфанда и Наймарка 1943 г. В случае банаховых алгебр обычно (явно или неявно) накладываются дополнительные условия, связывающие инволюцию и норму. Алгебра называется $C^{*}$-алгеброй, если $\left\|x x^{*}\right\|=\|x\|^{2}$ для всех ее элементов. В упомянутой работе установлено, что коммутативная $C^{*}$-алгебра естественно изоморфна алгебре всех непрерывных функций на своем пространстве максимальных идеалов.

Такие теоремы иногда имеют довольно неожиданные следствия. Скажем, из приведенной теоремы Гельфанда-Наймарка вытекает, что алгебра почти периодических функций Бора естественно изоморфна алгебре всех непрерывных функций на связной компактной группе $\left(\mathbb{R}_{d}\right)^{\wedge}$, где $\mathbb{R}_{\mathrm{d}}-$ вещественная ось в дискретной топологии, и отсюда легко выводится целый ряд фактов о почти периодических функциях Бора.

В дальнейшем Гельфанд и Наймарк установили, что каждая (т.е. не обязательно коммутативная) $C^{*}$-алгебра изоморфно реализуется в виде замкнутой самосопряженной подалгебры алгебры ограниченных операторов гильбертова пространства. Однако этому предшествовало исследование более общих алгебр с инволюцией, предпринятое Райковым. Одно из центральных мест в этом исследовании занимали положительные функционалы (для которых, по определению, $\left.\psi\left(x x^{*}\right) \geqslant 0\right)$. В случае групповых алгебр с естественной инволюцией $\left(f^{*}(x)=\overline{f(-x)}\right)$ имеется прямая связь между положительными функционалами и положительно-определенными функциями на группе, которая самим Д.А. использовалась для изучения групп (об этом уже было упомянуто выше).

Поворотным пунктом в развитии теории топологических групп считается работа Гельфанда и Райкова о бесконечномерных унитарных представлениях локально компактных групп. До этого полнота системы неприводимых представлений была установлена лишь для абелевых и компактных групп. Характерно, что в этих случаях неприводимые представления конечномерны. В то же время было известно, что существуют локально компактные группы, не имеющие таких представлений, кроме тривиального. До работы Гельфанда и Райкова полнота системы неприводимых унитарных представлений не была установлена даже для группы Лоренца.

Гельфанд и Райков, используя развитый Райковым аппарат положительноопределенных функций и теорему Крейна-Мильмана о крайних точках, доказали существование полной системы для каждой локально компактной группы. Судить о непосредственных результатах этого прорыва можно, например, по книгам [15] и [16].

\section{§ 6. Дополнительные примеры}

В заключение мы приведем несколько примеров, которые связаны с интересами Д.А. в гармоническом анализе. Характерная особенность, объединяющая эти примеры, состоит в том, что положительная определенность в конечном счете играет решающую роль, хотя априори речь о ней вроде бы не идет. 
Мы начнем с одного общего определения. Пусть $A$ - банахова алгебра с единицей $\mathbb{I}$ над полем $\mathbb{C}$ комплексных чисел. Линейный функционал $\psi: A \rightarrow \mathbb{C}$ называется состоянием, если $\|\psi\|=\psi(\mathbb{I})=1$. Множество всех состояний будем обозначать $\operatorname{St}(A)$. Легко видеть, что $\operatorname{St}(A)$ - выпуклый компакт в $\sigma\left(A^{*}, A\right)$-топологии.

Если $A$ есть $C^{*}$-алгебра, то линейный (непрерывный) функционал $\psi$, как мы отмечали, называется положительным, если $\psi\left(x^{*} x\right) \geqslant 0$ для всех $x \in A$. В случае $C^{*}$-алгебры функционал $\psi$ тогда и только тогда является положительным, когда $\|\psi\|=\psi(\mathbb{I})$. Поэтому положительный функционал в такой алгебре тогда и только тогда является состоянием, когда его норма равна 1.

Вернемся к общим банаховым алгебрам. Элемент $a \in A$ называется эрмитовым, если

$$
\psi(a) \in \mathbb{R} \quad \text { для всех } \quad \psi \in \operatorname{St}(A) .
$$

В случае $C^{*}$-алгебр эрмитовость равносильна самосопряженности $\left(a^{*}=a\right)$. Однако как в диск-алгебре, так и в алгебре абсолютно сходящихся рядов Фурье $L^{1}(\mathbb{Z})$ эрмитовы элементы сводятся к константам.

Элемент $x \in A$ называется разложимым, если он допускает представление $x=a+i b$, в котором $a$ и $b$ эрмитовы. Если такое представление существует, то оно единственно. В $C^{*}$-алгебре все элементы являются разложимыми.

Детально введенные понятия и история их возникновения обсуждаются в [17]. Там же можно найти доказательство теоремы Пальмера-Видава (T. W. Palmer, I. Vidav), придающей метрический характер теореме Гельфанда-Наймарка: если все элементы банаховой алгебры разложимы, то она является $C^{*}$-алгеброй относительно инволюции $a+i b=x \rightarrow x^{*}=a-i b$. Любопытно, что близкие соображения тесно связаны с исследованием ограниченных симметрических областей в бесконечномерных комплексных банаховых пространствах сравнительно новым направлением, развивающем исследования Э. Картана (см., в частности, [18]).

При $\sigma>0$ обозначим через $\mathbf{B}_{\sigma}$ совокупность всех целых функций экспоненциального типа не выше $\sigma$, ограниченных на вещественной оси $\mathbb{R}$. Из теоремы Фрагмена-Линделёфа вытекает, что для таких функций $f$ при всех $\lambda=\xi+i \eta \in \mathbb{C}$ выполняется неравенство

$$
|f(\lambda)| \leqslant e^{\sigma|\eta|} \cdot \max _{\mathbb{R}}|f(\xi)| .
$$

Отсюда следует, что $\mathbf{B}_{\sigma}$ - банахово пространство относительно поточечных операций и sup-нормы по вещественной оси. Из формулы Коши вытекает, что оператор $a=-i d / d \xi$ не выводит из пространства $\mathbf{B}_{\sigma}$. Легко убедиться, что спектр оператора $a$ в пространстве $\mathbf{B}_{\sigma}$ совпадает с отрезком $[-\sigma, \sigma]$, в частности, для его спектрального радиуса имеем $|a|=\sigma$.

Знаменитое неравенство С. Н. Бернштейна устанавливает, что

$$
\sup _{\mathbb{R}}\left|f^{\prime}(\xi)\right| \leqslant \sigma \sup _{\mathbb{R}}|f(\xi)| \text { для всех } f \in \mathbf{B}_{\sigma},
$$

и это означает, что $\|a\|=|a|$. Заметим, что $a$ является эрмитовым элементом алгебры всех ограниченных операторов пространства $\mathbf{B}_{\sigma}$ и что последнее 
равенство сохраняется для всех эрмитовых элементов всех банаховых алгебр. Прямое доказательство этого утверждения (и нескольких близких) имеется в [17].

С другой стороны, имеются многочисленные (в том числе многомерные) обобщения указанного неравенства Бернштейна. Классический подход к таким неравенствам в рамках теории целых функций, а также их приложения к обратным задачам теории приближений отражен в [19], [20].

Оказывается, в рамках абстрактного гармонического анализа часть из этих неравенств превращается в тривиальные [21]. Хотя результатам можно придать смысл в произвольных локально компактных абелевых группах, мы ограничимся здесь евклидовыми пространствами.

Пусть $Q$ - компакт в $\mathbb{R}^{n}$. Обозначим через $\mathbf{B}(Q)$ совокупность всех таких непрерывных и ограниченных функций на (двойственном экземпляре) $\mathbb{R}^{n}$, преобразование Фурье которых (понимаемое в смысле теории распределений Шварца) сосредоточено на $Q$. Относительно поточечных операций и sup-нормы по $\mathbb{R}^{n}$ множество $\mathbf{B}(Q)$ составляет банахово пространство. При $Q=[-\sigma, \sigma]$ получается $\mathbf{B}_{\sigma}$.

Если $f \in \mathbf{B}(Q)$ и $\mu \in M\left(\mathbb{R}^{n}\right)$, то $T_{\mu} f \in \mathbf{B}(Q)$, где $T_{\mu} f=f * \mu$ и

$$
(f * \mu)(\xi) \stackrel{\text { def }}{=} \int_{\mathbb{R}^{n}} f(\xi-\eta) \mu(d \eta) .
$$

Кроме того, $T_{\mu}=0$, если $\widehat{\mu}(\chi)=0$ в некоторой окрестности компакта $Q$. Имея это в виду, можно естественно расширить алгебру операторов $\left\{T_{\mu}\right\}$ до алгебры $\left\{T_{q}\right\}$, где $\{q\}$ - совокупность всех функций, локально совпадающих с функциями вида $\widehat{\mu}, \mu \in M\left(\mathbb{R}^{n}\right)$. Легко убедиться, что

$$
\operatorname{Spec}\left(T_{q}\right)=\{q(\chi) \mid \chi \in Q\}
$$

и что $\left\|T_{q}\right\|=\inf (\operatorname{var}(\nu))$, где нижняя грань берется по всем таким мерам $\nu$, для которых $\widehat{\nu}(\chi)=q(\chi)$ в некоторой окрестности компакта $Q$.

Теперь легко дать условия, по отдельности необходимые или достаточные для того, чтобы в $\mathbf{B}(Q)$ выполнялось равенство $\left\|T_{q}\right\|=\left|T_{q}\right|$. Для упрощения формулировок будем считать, что $0 \in Q$ и $1=q(0) \geqslant|q(\chi)|$ при всех $\chi \in Q$. Конечно, в таком случае $\left|T_{q}\right|=1$.

Необходимость: если $\left\|T_{q}\right\|=1$, то сужение $q \mid Q$ продолжается в $\mathbb{R}^{n}$ до функции из $\mathrm{PD}\left(\mathbb{R}^{n}\right)$. Заметим, что такое продолжение, вообще говоря, не единственно и среди таких продолжений не обязательно есть непрерывное.

Достаточность: $\left\|T_{q}\right\|=1$, если для каждого $\varepsilon>0$ существует такая мера $\nu \in$ $M\left(\mathbb{R}^{n}\right)$, что $\widehat{\nu}(x)=q(x)$ в некоторой окрестности компакта $Q$ и $\operatorname{var}(\nu)<1+\varepsilon$.

Есть такие классы компактов $Q$, что указанные условия смыкаются (отрезок на оси - один из простейших примеров).

Будем говорить, что компакт $Q$ удовлетворяет условию $(S)$, если $Q$ является множеством спектрального синтеза. Это означает, что если $f \in L^{1}\left(\mathbb{R}^{n}\right), \varepsilon>0$ и $\widehat{f} \mid Q=0$, то для подходящей функции $g \in L^{1}\left(\mathbb{R}^{n}\right)$ выполняются условия: $\|f-g\|<\varepsilon$ и $\widehat{g}(x)=0$ в некоторой окрестности компакта $Q$. Л. Шварц в 1948 г. показал, что единичная сфера $S^{2}$ в $\mathbb{R}^{3}$ не является множеством спектрального синтеза. 
Будем говорить, что компакт $Q$ удовлетворяет условию $(C)$ в точке $x_{0} \in Q$, если существует такое натуральное $m$, что для каждой окрестности $V$ точки $x_{0}$ множество

$$
\left\{ \pm x_{1}, \pm x_{2}, \ldots, \pm x_{m} \mid x_{1}, x_{2}, \ldots, x_{m} \in Q \cap V\right\}
$$

содержит некоторую окрестность точки 0 . Например, отрезок $[-\sigma, \sigma]$ на оси $\mathbb{R}$ и сфера положительного радиуса в $\mathbb{R}^{n}$ при $n \geqslant 2$ удовлетворяют условию $(C)$ в каждой своей точке.

Имеет место место следующий критерий. Предположим, что компакт $Q$ удовлетворяет условию $(S)$ и условию $(C)$ в точке 0 . В этом случае $\left\|T_{q}\right\|=1$ тогда и только тогда, когда $q \mid Q$ продолжается в $\mathbb{R}^{n}$ до непрерывной функции из $\mathrm{PD}\left(\mathbb{R}^{n}\right)$.

Среди прочего это позволяет обосновать пример Шварца. Действительно, пусть $Q=S^{2} \subset \mathbb{R}^{3}$. В пространстве $\mathbf{B}\left(S^{2}\right)$ рассмотрим оператор Лапласа

$$
\Delta=\partial^{2} / \partial t_{1}^{2}+\partial^{2} / \partial t_{2}^{2}+\partial^{2} / \partial t_{3}^{2} .
$$

Хорошо известно, что функция $\cos \sqrt{t_{1}^{2}+t_{2}^{2}+t_{3}^{2}}$ принадлежит пространству $\mathbf{B}\left(S^{2}\right)$. Поэтому $\|\Delta\|=3$, тогда как $\operatorname{Spec}(\Delta)=\{1\}$.

Теперь мы обсудим неравенство фон Неймана. Отметим, что наше рассуждение близко к тому, которое приводят Секефальви-Надь и Фояш [22; гл. 1 , $\S 8]$, но мы иначе расставляем акценты.

Классическое неравенство фон Неймана состоит в следующем. Пусть $T$ ограниченный оператор гильбертова пространства, причем $\|T\|<1$, и пусть $f-$ такой полином, что $|f(\lambda)|<1$ при $|\lambda|<1$. Тогда $\|f(T)\|<1$. В соответствии с теоремой Гельфанда-Наймарка оператор можно заменить таким элементом $a$ из $C^{*}$-алгебры $A$, для которого $\|a\|<1$, и утверждение будет состоять в том, что $\|f(a)\|<1$, и мы будем заниматься неравенством фон Неймана именно в этом варианте.

В случае $C^{*}$-алгебры $A$ мы пишем $x \geqslant 0$, если $x^{*}=x$ и $\operatorname{Spec}(x) \subset\{\lambda \mid \lambda \geqslant 0\}$. Легко проверить, что $x \geqslant 0$ тогда и только тогда, когда $\psi(x) \geqslant 0$ для всех $\psi \in \operatorname{St}(A)$.

Теперь мы перейдем непосредственно к неравенству фон Неймана. Легко показать, что общий случай сводится к случаю, когда $|a|<1$, где $|a|-$ спектральный радиус элемента $a$, и мы будем считать, что это условие выполнено. Отметим, что тогда $\overline{\lim }_{n \rightarrow \infty}\left\|a^{n}\right\|^{1 / n}<1$ и то же верно при замене $a$ на $a^{*}$.

При данном $a$ и данном $\psi \in \operatorname{St}(A)$ сформируем последовательность $\alpha \stackrel{\text { def }}{=}$ $\left\{\alpha_{n}, n \in \mathbb{Z}\right\}$ по следующему правилу: $\alpha_{0}=1$, а при целых $n \neq 0$ положим $\alpha_{n}=\psi\left(a^{n}\right)$, если $n>0$, и $\alpha_{n}=\overline{\alpha_{-n}}$, если $n<0$. Основное замечание состоит в том, что

$$
\|a\| \leqslant 1 \text { тогда и только тогда, когда } \alpha \in \mathrm{PD}(\mathbb{Z})
$$

(при каждом выборе $\psi \in \operatorname{St}(A))$.

Действительно, условие, касающееся $\alpha$, равносильно тому, что $\sum_{-\infty}^{\infty} \alpha_{n} \lambda^{n}$ $\geqslant 0$, если $|\lambda|=1$. Так как это должно иметь место при всех $\psi \in \operatorname{St}(A)$, то получается, что должно выполняться неравенство

$$
b \stackrel{\text { def }}{=}-\mathbb{I}+(\mathbb{I}-\lambda a)^{-1}+\left(\mathbb{I}-\bar{\lambda} a^{*}\right)^{-1} \geqslant 0
$$


(и обратно). Если $c$ - обратимый элемент алгебры $A$, то неравенство $b \geqslant 0$ равносильно неравенству $c b c^{*} \geqslant 0$. Если взять $c=\mathbb{I}-\lambda a$, то после очевидных сокращений получится, что исходное условие, касающееся $\alpha$, равносильно условию $\mathbb{I}-a a^{*} \geqslant 0$.

Пусть теперь $\|a\|<1$ и $f$ - такой полином, что $|f(\lambda)| \leqslant 1$ при $|\lambda| \leqslant 1$. Пусть $b=f(a)$ и $\psi \in \operatorname{St}(A)$. Обозначим через $\left\{\alpha_{n}\right\}$ и $\left\{\beta_{n}\right\}$ последовательности, отвечающие в указанном выше смысле элементам $a$ и $b$ соответственно.

По теореме Герглотца, существует такая вероятностная борелевская мера $\mu$ на единичной окружности $\mathbb{T}$, что

$$
\alpha_{n}=\int_{\mathbb{T}} \lambda^{n} \mu(d \lambda), \quad n \in \mathbb{Z} .
$$

Отсюда следует, что при целых $k>0$

$$
\beta_{k}=\int_{\mathbb{T}} f(\lambda)^{k} \mu(d \lambda)
$$

Так как $|f(\lambda)| \leqslant 1$, то отсюда легко следует, что $\left\{\beta_{n}\right\} \in \mathrm{PD}(\mathbb{Z})$.

В заключение мы опишем, как (среди прочего) установить, что $\zeta(1+i t) \neq 0$, используя только общие соображения, связанные с положительной определенностью (и эйлерово произведение $)^{7}$.

Предварительное замечание состоит в следующем. Пусть $X$ - локально компактная абелева группа и $f$ - вещественная непрерывная положительно-определенная функция на $X$. Обозначим через $f_{+}$функцию, совпадающую с $f$ там, где $f(x) \geqslant 0$, и равную 0 при всех остальных $x \in X$. Пусть $f_{-}=f-f_{+}$(так что $\left.f_{-} \geqslant 0\right)$. Замечание состоит в том, что если $f_{+} \in L^{1}(X)$, то и $f_{-} \in L^{1}(X)$, причем

$$
\int_{X} f_{-}(x) d x \leqslant \int_{X} f_{+}(x) d x
$$

(интеграл по мере Хаара). Если $f \in L^{1}(X)$, то функция $\widehat{f}$ на $G$ неотрицательна, непрерывна и суммируема, и формула для преобразований Фурье дает

$$
0 \leqslant \widehat{f}(0)=\int_{X} f(x) d x=\int_{X} f_{+}(x) d x-\int_{X} f_{-}(x) d x .
$$

В общем случае сначала "приглушается" рост $f$, а затем применяется какаянибудь теорема о предельном переходе под знаком интеграла Лебега.

Если $\sigma=\operatorname{Re} s>1$, то, используя эйлерово произведение, получаем

$$
\log \zeta(s)=\sum_{k, p} \frac{1}{k p^{s}} .
$$

Суммирование производится по всем натуральным $k$ и простым $p$. Из этого представления получается, что $g_{\sigma} \in \mathrm{PD}(\mathbb{R})$, где

$$
g_{\sigma}(t)=\log |\zeta(\sigma+i t)| \text {. }
$$

\footnotetext{
${ }^{7}$ В краткой форме я несколько раз рассказывал об этом. Подробное изложение см. в статье "Asymptotic law for the distribution of prime numbers in the context of free Abelian semigroups", Russ. J. Math. Phys., 13:1 (2006), 31-54.
} 
Пусть

$$
f(t)=\lim _{\sigma \rightarrow 1} g_{\sigma}(t) / g_{\sigma}(0)
$$

Легко видеть, что предел существует при всех $t \in \mathbb{R}$, причем $f(0)=1, f(t)=$ $k$, если $\zeta$-функция имеет нуль порядка $k$ в точке $1+i t$, и $f(t)=0$ при всех остальных $t \in \mathbb{R}$. В качестве поточечного предела функция $f$ остается в $\operatorname{PD}(\mathbb{R})$, хотя и теряет непрерывность. Рассматривая $\mathbb{R}$ в дискретной топологии, мы можем применить к $f$ сформулированное выше предложение. Так как $f-$ четная функция, то получается, что $\zeta(1+i t) \neq 0$.

Это рассуждение дословно распространяется на произведение классических $L$-функций Дирихле. Ввиду его полной прозрачности, оно приводит и к дальнейшим обобщениям как PNT, так и теоремы Дирихле о простых числах в арифметических прогрессиях. В частности, в сочетании с тауберовыми теоремами подобное рассуждение позволяет придать гораздо более законченный вид теоремам Б. М. Бредихина о базисных элементах свободных абелевых полугрупп (для полугрупп, удаленных от натурального ряда, у $\zeta$-функции может появиться конечное семейство нулей на прямой $\sigma=1$ и тогда в "асимптотическом законе" вместо константы возникает тригонометрический полином).

\section{Список литературы}

[1] Математика в СССР за сорок лет: 1917-1957, т. I, II, Физматгиз, М., Л., 1959.

[2] Юбилейный сборник $к$ 70-летию кафедры математического анализа МПГУ, МПГУ, М., 2004.

[3] Математика в СССР за тридцать лет: 1917-1947, Гостехиздат, М., Л., 1948.

[4] Математика в СССР 1958-1967, т. ІІ, вып. 2, Наука, М., Л., 1970.

[5] Ю.В. Линник, И.В. Островский, Разложение случайных величин и векторов, Наука, М., 1972.

[6] Л. З. Лившиц, И. В. Островский, Г. П. Чистяков, “Арифметика вероятностных законов", Итоги науки и техники. Теория вероятн. Матем. статист. Теорет. кибернет., 12, ВИНИТИ, М., 1975, 5-42.

[7] Е. Лукач, Характеристические функиии, Наука, М., 1979; E. Lukacs, Characteristic functions, Griffin, London, 1970.

[8] Г. М. Фельдман, Арифметика вероятностных распределений и характеризационные задачи на абелевых группах, Наукова думка, Киев, 1990.

[9] J. Korevaar, "A century of complex Tauberian theory", Bull. Amer. Math. Soc. (N.S.), 39:4 (2002), 475-531.

[10] Ю.А. Брудный, "О локальном наилучшем приближении функций многочленами", Докл. АН СССР, 161:4 (1965), 746-749.

[11] Э. Хьюитт, K. Росс, Абстрактный гармонический анализ. Т. II: Структура и анализ компактных групп. Анализ на локально компактных абелевых группах, Мир, М., 1975; Abstract harmonic analysis. V. II: Structure and analysis for compact groups. Analysis on locally compact Abelian groups, Grundlehren Math. Wiss., 152, Springer-Verlag, New York, 1970.

[12] E. A. Gorin, "Universal symbols on locally compact abelian groups", Bull. Polish Acad. Sci. Math., 51:2 (2003), 199-204.

[13] И. М. Гельфанд, Д. А. Райков, Г.Е. Шилов, Коммутативные нормированные колъца, Физматгиз, М., 1960. 
[14] J. L. Taylor, "The cohomology of the spectrum of a measure algebra", Acta Math., 126:1 (1971), 195-225.

[15] М.А. Наймарк, Нормированные кольц, Наука, М., 1968.

[16] А.А. Кириллов, Элементы теории представлений, Наука, М., 1978.

[17] F.F. Bonsall, J. Duncan, Complete normed algebras, Ergeb. Math. Grenzgeb., 80, Springer-Verlag, New York, 1973.

[18] W. Kaup, "A Riemann mapping theorem for bounded symmetric domains in complex Banach spaces", Math. Z., 183:4 (1983), 503-529.

[19] Н. И. Ахиезер, Избранные труды по теории функиий и математической физике, т. I, II, АKTA, Харьков, 2001.

[20] B. Ya. Levin, Lectures on entire functions, Transl. Math. Monogr., 150, Amer. Math. Soc., Providence, RI, 1996.

[21] Е.А. Горин, "Неравенства Бернштейна с точки зрения теории операторов", Вестн. Харък. ун-та. Сер. прикл. матем. и мех., 205:45 (1980), 77-105; E. A. Gorin, "Bernstein's inequality from the point of view of operator theory", Selecta Math. Sov., 7:3 (1988), 191-219.

[22] Б. Секефальди-Надь, Ч. Фояш, Гармонический анализ операторов в гильбертовом пространстве, Мир, М., 1970; B. Sz.-Nagy, C. Foiaş, Analyse harmonique des opérateurs de l'espace de Hilbert, Masson, Paris, 1967.

Е. А. Горин (Е. А. Gorin)

Московский педагогический государственный университет

E-mail: evgeny.gorin@mtu-net.ru
Поступила в редакцию

17.01 .2006 